\title{
Finite Element Stress Analysis of a Restored Axisymmetric First Molar
}

\author{
J. W. FARAH and R. G. CRAIG \\ University of Michigan, School of Dentistry, Ann Arbor, Michigan 48104, USA
}

\begin{abstract}
A first molar with full crown preparation and three marginal configurations - chamfer, chisel, and shoulder with a bevel-was analyzed by the finite element method. The maximum, minimum, and shear stresses were plotted as a function of the radius along various horizontal planes. The chamfer geometry exhibited the most uniform stress distribution.
\end{abstract}

Experimental stress analysis of dental structures has been a topic of interest during the past quarter century. The object of such research was the determination of stress distribution and improvement of the mechanical strength of these structures. Most of the stress analysis of dental structures was carried out using the photoelastic technique. ${ }^{1-8}$

A more recent method of stress analysis, originally developed in $1956^{9}$ in the aircraft industry, is the finite element method. This technique has since been in widespread use not only in aerospace engineering, but also in civil engineering. The finite element method has proved to be extremely effective for the treatment of problems of plane stress and plane strain. 10,11

The purpose of this study was to examine the distribution of the stresses in a first molar with three marginal configurations using the finite element method.

\section{Materials and Methods}

The concept of the finite element method involves the idealization of the actual con-

This investigation was supported by USPHS Research Grant DE-01817 from the National Institute of Dental Research, National Institutes of Health, Bethesda, Md.

This paper was presented at the 51 st general session of the IADR in Washington, DC, April 1973.

This paper was based on a portion of a dissertation in partial fulfilment of the $\mathrm{PhD}$ degree in dental materials and aerospace engineering (mechanics) at the University of Michigan, 1972.

Received for publication May 25, 1973. tinuum (or model) as an assemblage of a finite number of discrete structural elements interconnected at a finite number of points or nodal points. The finite elements are formed by figuratively cutting the original continuum into a number of appropriately shaped sections and retaining in the elements the properties of the original material. The analysis process consists of satisfying compatibility within each element and equilibrium conditions at the nodal points. By concentrating the equivalent forces at the nodes, equilibrium conditions are satisfied in the overall sense only. Local violations of equilibrium conditions within each element and at its boundaries could arise. The finite element appoach is, in a sense, equivalent to the minimization of the total potential energy of the system in terms of a prescribed displacement field. If the displacement field is defined in a suitable way, then convergence to the correct value will occur.

A computer program was written to evaluate the stresses in the axisymmetric model. The following information is needed for calculating the stresses:

1. the total number of nodal points,

2. the total number of elements,

3. a numbering system identifying each element,

4. the Young's modulus and Poisson's ratio associated with each element,

5. a numbering system identifying each nodal point,

6. the coordinates of each nodal point,

7. the type of boundary constraints, and

8. the evaluation of the forces at the external nodes.

After these data are specified, the displacements, as well as the stresses, can be calculated immediately with the program. The program was checked for the case of a thickwalled cylinder under internal pressure, and 
the results of the stresses obtained by the finite element method agreed remarkably well 12 with the theoretical values given by Timoshenko and Goodier. ${ }^{13}$

The axisymmetric model of an idealized first molar was subdivided into 348 triangular elements, and a number was assigned to each element as shown in Figure 1. The model was loaded with an axisymmetric load of 100 $\mathrm{lb}$, and it was assumed that the model was fixed at the base. The three marginal configurations investigated were: a shoulder with a bevel, a chamfer, and a chisel, or feather-edge. Also, the effects on the stresses by the introduction of a pulp chamber were examined. The three marginal configurations, as well as the pulp chamber, are shown in Figure 1. It also was assumed that for each marginal configuration the enamel was completely removed, and the dentin and gold were isotropic and homogeneous. The principal stresses, as well as the maximum shear stresses at the centroid of each triangular element, were calculated using the following material properties: (1) a modulus of elasticity for gold of $13 \times 10^{6}$ psi and a Poisson's ratio of 0.33 ; (2) a modulus of elasticity for dentin of $2.7 \times 10^{6} \mathrm{psi}$ and a Poisson's ratio of 0.31 ; and (3) a modulus of elasticity for the pulp of 300 psi and a Poisson's ratio of 0.45. The modulus and Poisson's ratio chosen for the pulp are estimates or probable values and are used only to facilitate the operation of the computer program.

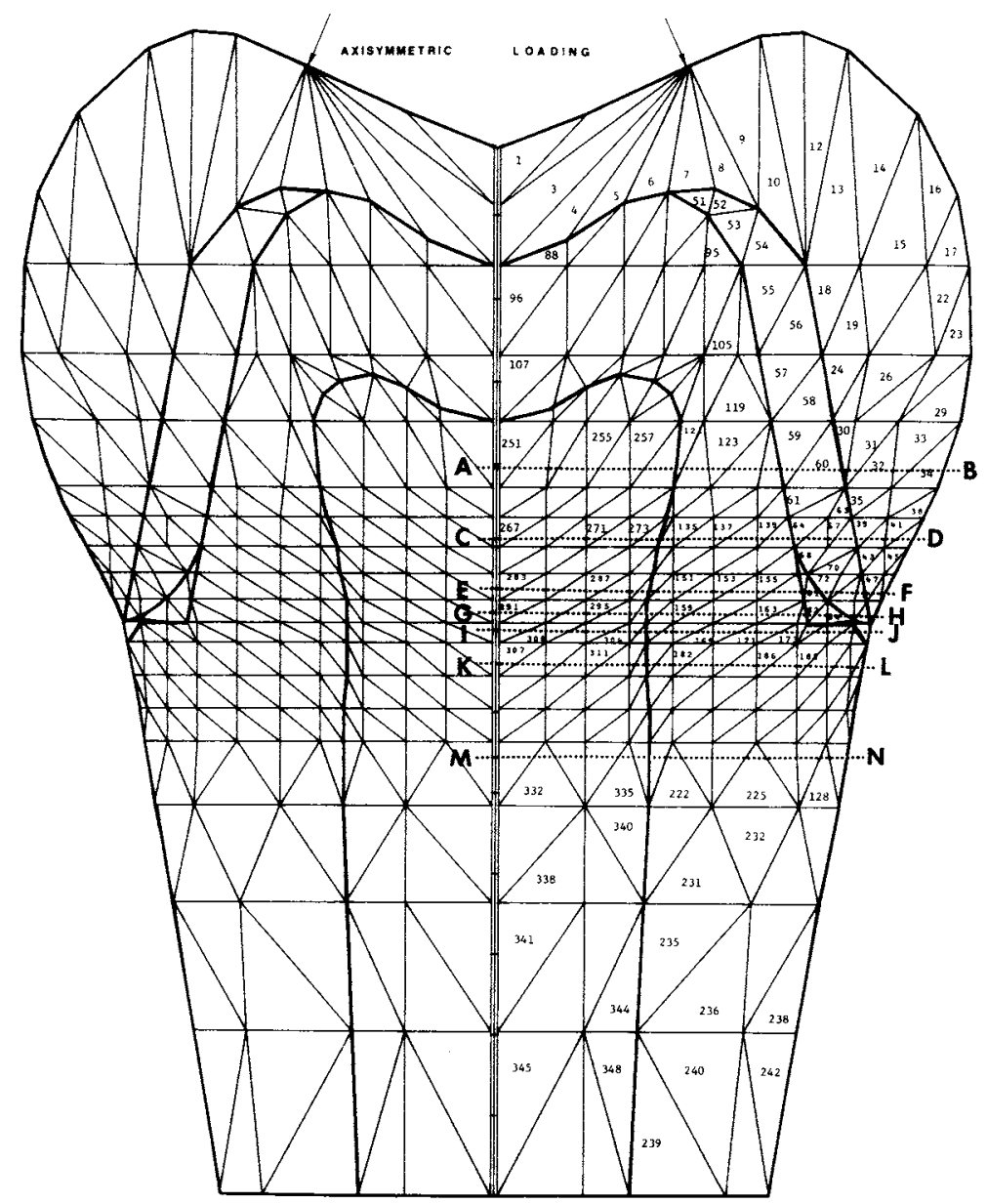

FIG 1.-Axisymmetric finite element model with three marginal configurations. 


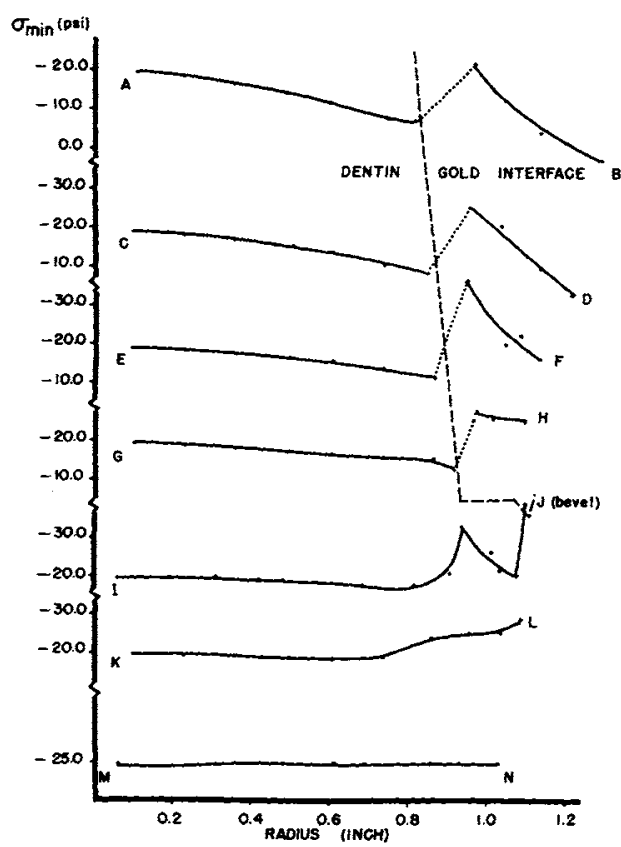

FIc 2.-Minimum axial stress, $\sigma_{m t n}$, as a function of radius along specified planes for shoulder geometry.

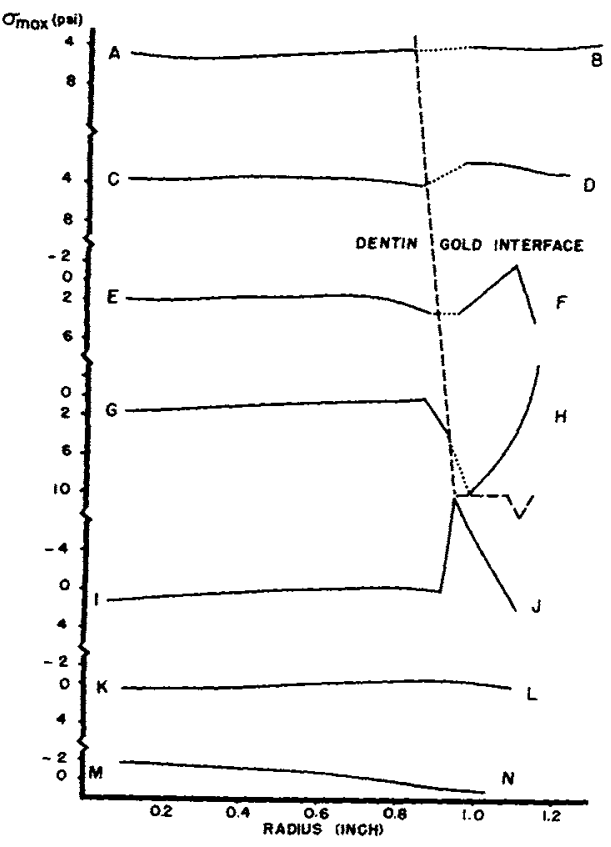

Fig 3.- Maximum radial stress, $\sigma_{\text {max }}$ as a function of radius along specified planes for shoulder geometry.

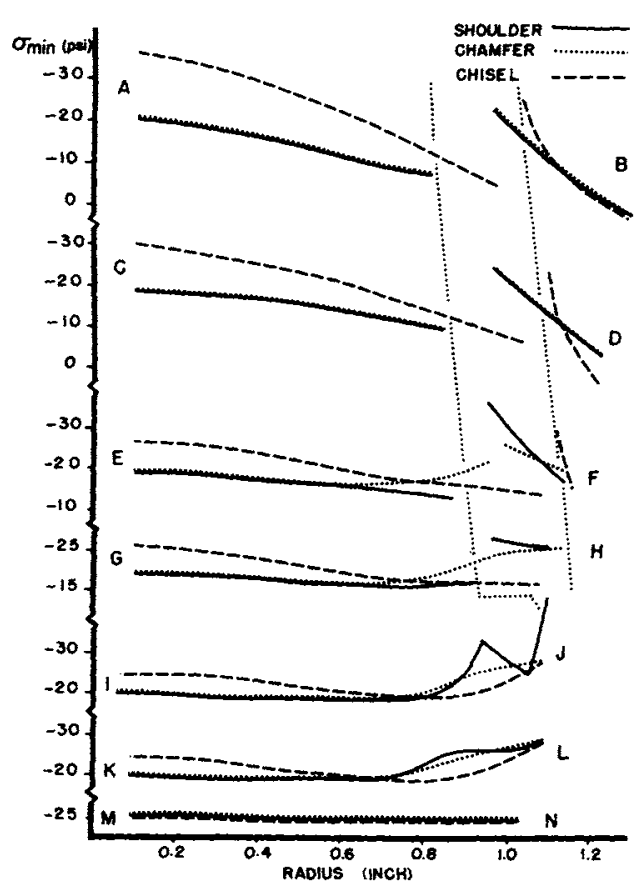

Fig 4.-Comparison of minimum axial stress, $\sigma_{m ! n}$, for the three marginal configurations without pulp preparation.

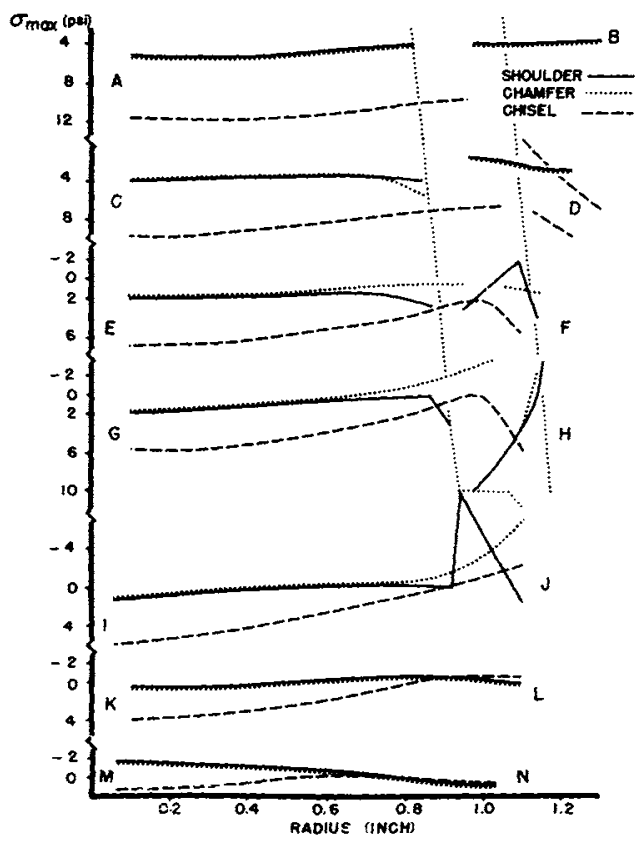

Fig 5.-Comparison of maximum radial stress, $\sigma_{\text {max }}$, for the three marginal configurations without pulp preparation. 


\section{Results}

The minimum axial stresses, $\sigma_{\text {min }}$, the maximum radial stresses, $\sigma_{\max }$, as well as the maximum shear srtesses, $\tau_{\text {max }}$, along the planes $A-B$ through $M-N$ are plotted in Figures 2 through 6 where the pulp chamber was not considered; that is, it was assumed that dentin occupied that area. Figures 2 and 3 show the distribution of $\sigma_{\min }$ and $\sigma_{\max }$ along the specified lines for the shoulder geometry. The dotted lines simply connect stress values in the same plane on either side of the dentin-gold interface. In Figures 4,5 , and $6, \sigma_{\min }, \sigma_{\max }$, and $\tau_{\max }$ of the three marginal configurations are superimposed and compared.

The stresses $\sigma_{\min }$ and $\tau_{\max }$ are plotted in Figures 7 and 8 for the model that has an idealized pulp chamber (see Figure 1).

The axisymmetric model was scaled down, by a factor of 6.3 , to the size of an actual molar, and the stresses $\sigma_{\min }, \sigma_{\max }$, and $\tau_{\max }$ were determined.

The stresses $\sigma_{\min }$ for the scaled-down model are plotted and compared with the stresses $\sigma_{\min }$ in the actual-size model in Figure 9.

\section{Discussion}

The introduction of the idealized axisym-

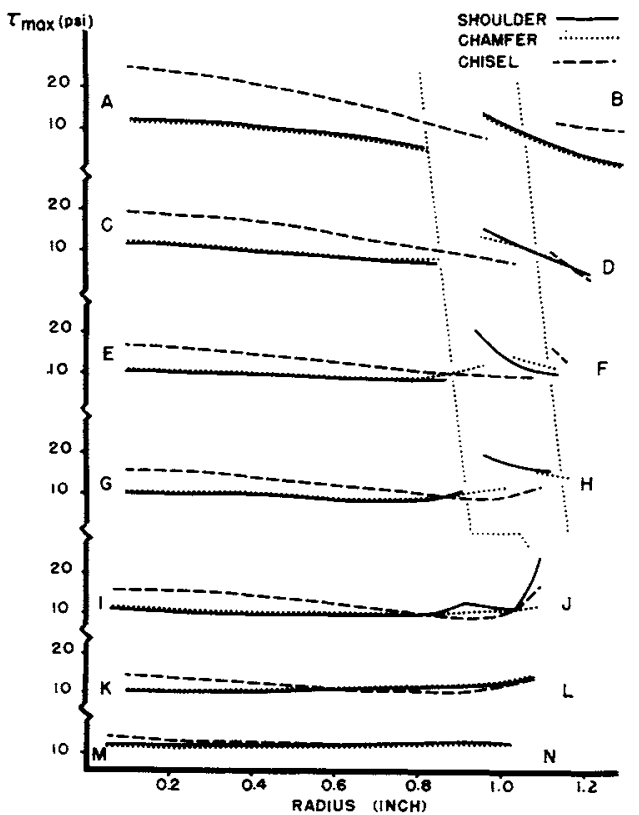

FIG 6.-Comparison of maximum shear stress, $\tau_{\max }$, for the three marginal configurations with. out pulp preparation.

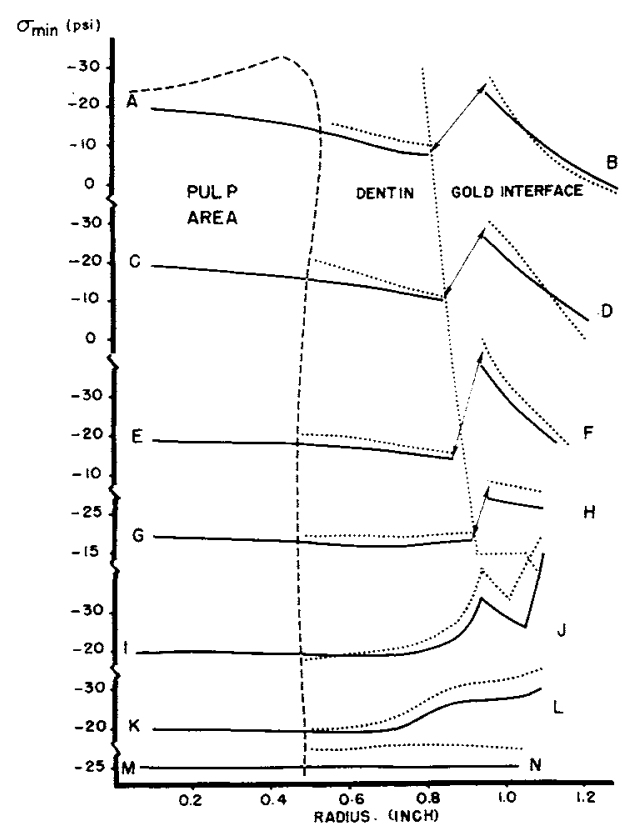

FIG 7.-Minimum axial stress, $\sigma_{m i n}$, for shoulder geometry with pulp preparation. Solid lines indicate stresses in model without pulp; dotted lines indicate stresses in model with pulp.

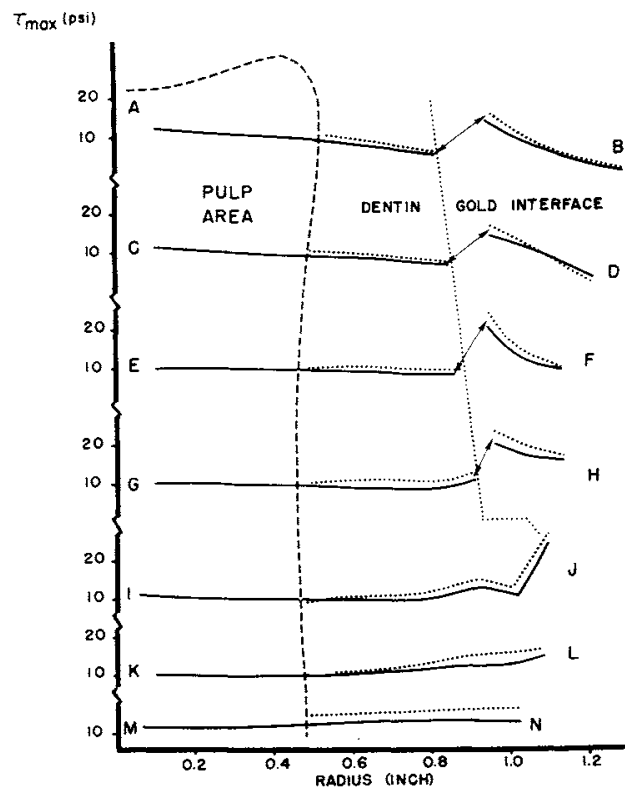

Fic 8.-Maximum shear stress, $\tau_{\max }$, for the shoulder geometry with pulp preparation. Solid lines indicate stresses in model without pulp; dotted lines indicate stresses in model with pulp. 

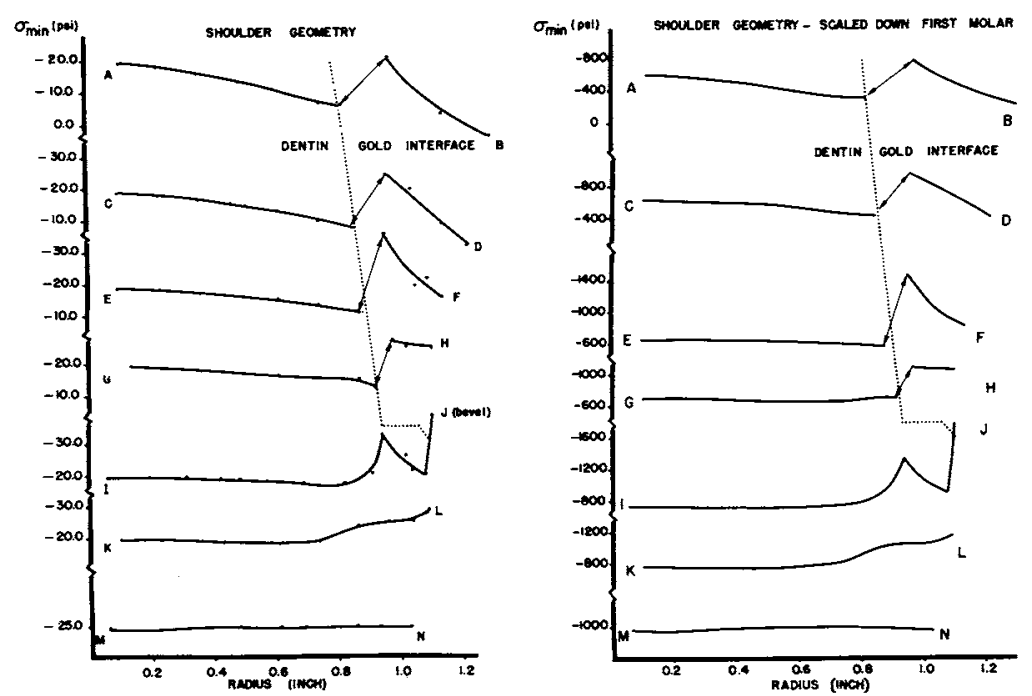

Fig 9.-Comparison of principal axial stress, $\sigma_{m ! n}$, in actual size model to scale-down model.

metric model is based on some of the results obtained earlier ${ }^{14}$ on actual composite photoelastic three-dimensional models. Sectioning of the photoelastic models revealed that the shear stress distribution was fairly symmetrical on both sides of a plane passing through the middle of the molar. Symmetry in the shear stress also was observed in slices made along the long axis of the tooth.

In Atlas on Tooth Form, Wheeler showed 15 that the diameter at the cervix measured mesiodistally or buccolingually was the same. Furthermore, the diameter of the crown of the mandibular first molar was also quite similar whether measured mesiodistally or buccolingually. These observations led to the introduction of the first molar as an axisymmetric idealization.

With the introduction of the axisymmetric model, a decision had to be made as to the number of elements needed to obtain reliable and consistent information pertaining to the stresses. In one instance the axisymmetric model was subdivided into 154 elements, in another into 348 elements, and in a third into 701 elements. The stresses obtained in the first instance were within $10 \%$ of those in the second. However, as the number of elements was increased to 701 , the difference in the stress between the second and third instances was negliglible. This is an encouraging aspect of the finite element method, and it proved that reliable information can be obtained without using an excessively large number of elements.

In Figure 2 the variation in $\sigma_{\min }$ along lines $A-B$ through $M-N$ for the beveled shoulder geometry is shown. The $\sigma_{\min }$ stresses along the plane $M-N$ had a constant value, which demonstrated that they varied little the further away the area was from the point of application of the load. In the proximity of the shoulder, the stress $\sigma_{\min }$ increased from about -19 psi to more than -33 psi on line $I-J$. Lines $A-B$ through $G-H$ exhibit the same trends of stress distribution; that is, the compressive axial stresses were fairly constant throughout the dentin and in areas away from the dentin-gold interface. They decreased moderately as the dentin-gold interface was approached. Higher stresses were encountered in the gold portion of the model because of the higher physical properties of the gold.

Figure 4 compares the minimum axial stresses of the three marginal configurations. Again the stresses along plane $M-N$ for the three geometries were constant and of the same magnitude. The lack of a sharp increase in stress along line $I-J$ of the chamfer and chisel edge geometry was noticed. Also, along lines $A-B$ through $G-H$ the stresses in the three geometries followed the same trend. The higher stresses of the chisel edge geometry should be observed. Such findings imply 
that the gold crowns acted as a strengthening mechanism to the dentin, and since less gold was used in the chisel edge geometry, the structure was weaker, and therefore the stresses were higher. It should be remembered that in each of these instances it was assumed that the enamel was completely removed. If the area between the margin of the shoulder and the margin of the chisel edge (Fig I) had been replaced with enamel instead of dentin, the stress distribution of the three geometries would have had similar trends and been of the same magnitude, except in the immediate vicinity of the shoulder; this is due to the fact that the modulus of the enamel as reported by Craig, Peyton, and Johnson, ${ }^{16}$ and Hall et al17 is approximately equal to the modulus of gold. Craig, Peyton, and Johnson reported the modulus to be about $12 \times 10^{6}$ psi, whereas $\mathrm{Hall}$ et al put it in the range from $11-12 \times 10^{6} \mathrm{psi}$. In the first instance, the modulus was found by means of static mechanical testing, whereas in the latter ultrasonic techniques were used.

Similar observations can be made from Figures 3 and 5 , where the maximum radial stress was plotted as a function of the radius. The discontinuity across the dentin-gold interface was of a lesser magnitude because equilibrium had to be satisfied in the radial direction. An exception was observed in line $G-H$ where the radial stress was quite different at the dentin-gold interface. This exception is a result of the proximity of line $G-H$ to the shoulder, that is, of right angle geometry.

Pronounced changes in the maximum radial stress from tension to compression were noticed in areas near the shoulder and the dentin-gold interface, and it is believed that smaller subdivisions of the elements in that area would be necessary to obtain a more precise measure of the stress.

A constant value of the maximum shear stress along line $M-N$ was observed (Fig 6). The $\tau_{\max }$ stresses followed the same trends as $\sigma_{\min }$ stresses as explained previously with regard to Figure 2.

In Figures 7 and 8 the $\sigma_{\min }$ and $\tau_{\max }$ stresses were plotted as a function of the radius for the axisymmetric model with the shoulder geometry and with a pulp chamber. In both instances the stresses followed much the same pattern, except, as would be expected, they were slightly higher for the model of the molar with the pulp chamber.
Finally, the idealized axisymmetric model was scaled-down to the size of an actual molar, and the maximum axial stresses were plotted and compared to the maximum axial stresses in the oversized model (Fig 9). The scaling was done in order to draw some conclusions and correlations between the oversized model and an actual molar subjected to the same loading conditions. In scaling, the radial coordinates $(r, z)$ of the oversized model were divided by a constant, in this instance equal to 6.3 , which was equal to the number of times the original model was amplified. The original model was oversized to relate the stresses obtained by the finite element method to those obtained by the method of three-dimensional photoelasticity. 14 The scaling-down did not alter the trends of the stress distribution, but the magnitude of the stresses was forty times higher. The magnitude as well as the direction of the stress at the dentin-gold interface is of great interest, because this knowledge would help determine the kind of cement to be used or could provide the reason why failure might occur at the interface.

The maximum shear stresses of the scaleddown model, having the shoulder geometry in areas near the dentin-gold interface, varied from 138 to $487 \mathrm{psi}$ in the dentin and from 505 to $974 \mathrm{psi}$ in the gold crown (Table). The higher values of $\boldsymbol{\tau}_{\max }$ were located in each instance in the region where a sudden change in geometry occurred. These values of the shear stress at the dentin-gold interface are interesting in view of the fact that the tensile and shear strengths of dental cements fall in the range of 200 to 1,000 psi. The cement is, therefore, the weak link in any cemented dental restoration and any failure will most likely be caused by it. Also, when examining the stresses in adjacent elements on either side of the dentin-gold interface, a rather steep stress gradient was observed. For example, along line $C-D$ the axial stress, $\sigma_{\max }$, for elements 140 and 64 was approximately 110 and -90 psi and for elements 165 and 82 along line G.H the axial stress was 110 and -105 psi, respectively. Higher stress gradient values occurred at the shoulder, that is, at the area where the geometry changed most rapidly. These high radial stress gradients combined with the maximum values of the shear stress could cause failure of a restoration because of failure in the cement. 
TABLE

Stresses at the Dentin-Gold InTERface in the SCaled-Down Model

\begin{tabular}{crrrrrrr}
\hline $\begin{array}{c}\text { Dentin } \\
\text { Element }\end{array}$ & \multicolumn{1}{c}{$\sigma_{\text {max }}$} & \multicolumn{1}{c}{$\sigma_{\text {m In }}$} & $\tau_{\text {max }}$ & $\begin{array}{c}\text { Gold } \\
\text { Element }\end{array}$ & $\sigma_{\text {max }}$ & $\sigma_{\text {m In }}$ & $\tau_{\text {max }}$ \\
\hline 95 & -2.3 & -485 & 241 & 53 & 108 & $-1,777$ & 942 \\
106 & 9.8 & -308 & 159 & 55 & -17 & $-1,463$ & 723 \\
120 & 46.0 & -230 & 138 & 57 & -73 & $-1,178$ & 553 \\
125 & 89.0 & -343 & 216 & 59 & -98 & $-1,117$ & 510 \\
132 & 129.0 & -336 & 233 & 61 & -106 & $-1,116$ & 505 \\
140 & 110.0 & -445 & 277 & 64 & -87 & $-1,164$ & 539 \\
148 & 148.0 & -453 & 301 & 79 & 39 & $-1,362$ & 700 \\
156 & 80.0 & -581 & 330 & 80 & -54 & $-1,504$ & 725 \\
165 & 110.0 & -746 & 428 & 82 & -104 & $-1,738$ & 817 \\
175 & -432.0 & $-1,360$ & 464 & 83 & 367 & $-1,162$ & 764 \\
177 & -264.0 & $-1,094$ & 415 & 84 & 295 & $-1,057$ & 676 \\
179 & 31.0 & -943 & 487 & 87 & 135 & $-1,812$ & 974
\end{tabular}

In the chamfer geometry model, the radial stress gradients would be of small magnitude. The axisymmetric model is an idealized model considered under ideal loading conditions. Under these idealized conditions, the chamfer geometry exhibited the most acceptable trends of stress distribution, that is, it lacked acute stress concentration areas, followed by the shoulder and the chisel edge configuration. It also was shown that even under these idealized loading conditions the maximum shear stresses were equivalent to or greater than the maximum tensile and shear strength of most dental cements. Since ideal conditions, such as complete symmetry in geometry and loading, rarely occur in the oral environment, it could be concluded that far more critical stress concentrations can occur in the mouth, causing the cement to fail and the subsequent failure of the restoration.

\section{Conclusions}

The use of an idealized axisymmetric model proved to be a sound approach for obtaining approximate, yet reliable, information concerning the stress distribution in a dental model with different marginal configurations. Compared with the photoelastic or strain gauge methods, the finite element method has the advantage of providing a detailed evaluation of the complete state of stress in the dental model. A relatively small number of elements strategically located can provide sufficient yet accurate information concerning the state of stress in the model. It was found that the change in marginal configuration of the crown affected the stress distribution only in regions in the immediate vicinity of such change. The chamfer geometry exhibited the least amount of axial, radial, and shear stress at the margin, followed by the shoulder and the chisel edge geometries, both of which did not differ much except at points immediately under the shoulder. Furthermore, the maximum shear stress in the actual size model at the dentin-gold interface ranged from 138 to 487 psi in the dentin and 505 to 974 psi in the gold. This shear stress is of the same magnitude of most cements used in dentistry and suggests the possibility of failure of the cement at the interface. Moving from the dentin to the gold, the maximum radial stress gradient varied from values as low as $9.8 \mathrm{psi}$ in the dentin and -17 psi in the gold to as high as $-432 \mathrm{psi}$ in the dentin and $367 \mathrm{psi}$ in the gold in adjacent elements. Such large radial stress gradients will contribute to the failure of the cement, since dental cements are relatively weak in tension.

In an idealized model, having a symmetrical geometry and well-distributed loading, changes in the marginal configuration have little effect on the stress distribution as a whole, but significant stress gradients occur at the dentin-gold interface which could cause failure of a restoration because of failure of the cement.

\section{References}

1. Noonan, M.A.: The Use of Photoelasticity in a Study of Cavity Preparations, $J$ Dent Child 16: 24-28, 1949.

2. Haskins, R.C.; HaACK, D.C.; and Ireland, R.L.: A Study of Stress Pattern Variation in Class II Cavity Restorations as a Result of Different Cavity Designs, J Dent Res 33: 757 . 766, 1954. 
3. Mahler, D.B., and Peyton, F.A.: Photoelasticity as a Research Technique for Analyzing Stresses in Dental Structures, $J$ Dent Res 34: 831-838, 1955.

4. Lehman, M.L., and Hampson, E.L.: A Study of Strain Patterns in Jacket Crowns on Anterior Teeth Resulting from Different Tooth Preparations, $\mathrm{Br}$ Dent $J$ 113: 337-345, 1962.

5. Johnson, E.W.: A Three-dimensional Photoelastic Investigation of Stress Concentrations in Operably Deformed Human Teeth, thesis, University of Alberta, School of Engineering, 1965.

6. Johnson, E.W.; Castaldi, C.R.; Gau, D.J.; and WYsocki, G.P.: Stress Pattern Variations in Operatively Prepared Human Teeth, Studied by Three-dimensional Photoelasticity, J Dent Res 47: 548-558, 1968.

7. Craig, R.G.; El-ebrashi, M.K.; and Peyton, F.A.: Experimental Stress Analysis of Dental Restorations, II. Two-dimensional Photoelastic Stress Analysis of Crowns, $J$ Prosthet Dent 17: 292-302, 1967.

8. Ell-ebrashi, M.K.; Craig, R.G.; and Peyton, F.A.: Experimental Stress Analysis of Dental Restorations, III. The Concept of the Geometry of Proximal Margins, J Prosthet
Dent 22: 333-345, 1969.

9. Turner, M.J.; Clough, R.W.; Martin, H.C.; and TOPP, L.J.: Stiffness and Deflection Analysis of Complex Structures, $J$ Aero $S_{c i} 23$ : 805-823, 1956.

10. Clough, R.W.: The Finite Element Method in Plane Stress Analysis, Proceedings of the 2nd ASCE Conference on Electronic Computation, Pittsburgh, September 1960.

11. Clough, R.W., and Wilson, E.C.: Stress Analysis of a Gravity Dam by the Finite Element Method, RILEM Bull 19:45-54, 1963.

12. FARAH, J.W.: Stress Analysis of First Molars with Full-crown Preparations by Three-dimensional Photoelasticity and the Finite Element Method, PhD dissertation, University of Michigan, 1972.

13. Timoshenko, S., and Goodier, J.N.: Theory of Elasticity, New York: McGraw-Hill, 1951.

14. FARAH, J.W., and CRAIG, R.G.: Three-dimensional Photoelastic Stress Analysis of First Molars with Full-crown Preparations, IADR Program and Abstracts of Papers, No. 864, 1972.

15. WheEler, R.C.: An Atlas of Tooth Form, Philadelphia: W. B. Saunders Co., 1969. 